07

\title{
Управляемый логический вентиль на четырехузловом линейном гибридном кластерном состоянии
}

\author{
(C) К.С. Тихонов ${ }^{1}$, А.Д. Манухова ${ }^{2}$, С.Б. Королёв ${ }^{1}$, Т.Ю. Голубева ${ }^{1}$, Ю.М. Голубев ${ }^{1}$ \\ ${ }^{1}$ Санкт-Петербургский государственный университет, \\ 199034 Санкт-Петербург, Россия \\ ${ }^{2}$ Palacky University, Department of Optics, \\ 77146 Olomouc, Czech Republic \\ e-mail: tikhonov.kyril@gmail.com
}

Поступила в редакцию 08.04.2019 г.

В окончательной редакции 08.04.2019 г.

Принята к публикации 24.04.2019 г.

В случае большого количества вычислений может возникнуть ситуация, когда промежуточные результаты вычислений необходимо хранить некоторое заданное время. В этой работе показано, как создать гибридное атомно-полевое кластерное состояние и реализовать управляемый вентиль $C Z$ таким образом, чтобы результаты вычисления оказались записанными на долгоживущих степенях свободы атомного ансамбля и могли сохраняться продолжительное время.

Ключевые слова: однонаправленные вычисления, кластерное квантовое состояние, квантовая память, управляемый логический вентиль.

DOI: $10.21883 /$ OS.2019.11.48520.141-19

\section{1. Введение}

Идея однонаправленных квантовых вычислений возникла при поиске новых подходов к созданию универсального квантового компьютера [1]. В отличие от стандартной модели квантовых вычислений на обратимых логических вентилях [2] однонаправленные квантовые вычисления предполагают выполнение цепочки локальных проекционных измерений над элементами многочастично запутанной физической системы, находящейся в кластерном состоянии. Физическая система в кластерном состоянии при таком подходе выступает в роли своеобразного „ресурса“, который расходуется по мере выполнения вычислений (измерений): происходит поэтапная редукция квантовой запутанности. Естественно, такой процесс является необратимым, однонаправленным, и для выполнения новых вычислений необходимо заново приготовить систему в кластерном состоянии. Несмотря на это, было показано, что по вычислительным возможностям однонаправленные квантовые вычисления не уступают стандартной модели на обратимых логических вентилях [3].

Реализация квантовых вычислений в непрерывных переменных [4] на сегодня привлекает все большее внимание исследователей как интересная альтернатива концепции вычислений в дискретных переменных [2]. При этом, несмотря на видимую аналогию, принципы вычислений в дискретных и непрерывных переменных, а соответственно и протоколы их реализации существенно отличаются. Основным отличием квантовых вычислений на физических системах, описываемых непрерывными переменными, от дискретных является то, что для первых все квантовые логические операции носят детерминистический, а не вероятностный характер. Для осуществления однонаправленных вычислений в непрерывных переменных достаточно гомодинного детектирования, посредством которого проводят цепочку проекционных измерений над отдельными элементами системы (осцилляторами) в кластерном состоянии [5]. Классическая линия связи позволяет учесть результат предыдущих этапов измерений при выполнении новых.

В настоящее время активно ведется поиск физических систем, удобных для осуществления вычислительных операций. Наиболее распространенной системой здесь являются световые кластеры [6], разрабатываются также подходы, использующие многомодовую запутанность спиновых волн внутри атомных ансамблей [7,8], собственных мод оптомеханических систем [9]. Световые кластеры наиболее просты с точки зрения генерации и детектирования, такие состояния реализованы экспериментально на основе последовательности сжатых импульсов [10]. Однако световые системы трудны в использовании, когда требуется ввести временную задержку между операциями. Такая ситуация может возникать, когда выполняемая операция зависит от результата другого вычисления, еще не полученного. Или же в случае, если вычислительная система делокализована, и необходимо дождаться, пока часть узлов будет переправлена на значительные расстояния. Эту проблему решает использование так называемых гибридных кластерных состояний. В работе [8] предложена схема реализации таких состояний с помощью QND-взаимодействия атомных ячеек со световыми полями. 
Здесь мы рассмотрим другой подход к формированию атомно-полевого кластерного состояния. Сначала сформируем чисто полевое кластерное состояние на основе квадратурно-сжатых световых импульсов, а уже затем „отпечатаем“ требуемые узлы на долгоживущие степени свободы атомного ансамбля внутри ячейки квантовой памяти. Как будет показано ниже, такой тип состояния позволяет выполнить операцию двухмодового управляемого вентиля таким образом, чтобы результат вычисления оказался сохраненным внутри ячеек памяти. С одной стороны, это обеспечивает схему всеми достоинствами легкого ввода и вывода сигнала, над которым производится преобразование. С другой, сформированный таким образом гибридный кластер позволяет проделывать делокализованные вычисления.

Мы продемонстрируем работу такой системы на примере управляемого вентиля $C Z$. Квантовый компьютер для успешной работы должен выполнять любое требуемое преобразование или, говоря иначе, универсальное преобразование. Для квантовых компьютеров на непрерывных переменных эта универсальность сводится к способности реализовать два основных типа операций: универсальных многомодовых гауссовых и одномодовой негауссовой [4]. Гауссовым квантовым преобразованием называется преобразование, которое изменяет входное состояние линейным образом, а негауссовым - нелинейное преобразование. Таким образом, если для какойлибо системы удается продемонстрировать, что с ее помощью возможно выполнить оба указанных выше типа преобразований, то можно утверждать, что на такой системе возможно осуществить любую логическую операцию. В этой работе мы, соответственно, остановимся на первом типе преобразований - гауссовом многомодовом, беря за основу подход, разработанный в [11] для светового кластера. Мы покажем, что включение в схему элементов с потерями требует определенной симметризации и предложим вариант ее реализации.

Работа организована следующим образом. В разд. 2 вводятся основные понятия, необходимые для описания кластерного состояния квантовой системы в непрерывных переменных. Следующий раздел посвящен описанию процедуры формирования гибридного кластерного состояния. В разд. 4 мы показываем, что наличие несимметричных потерь разрушает кластер, и предлагаем схему симметризации потерь. Раздел 5 посвящен реализации вентиля $C Z$ на полученном гибридном кластерном состоянии. В заключении мы обсуждаем возможные пути оптимизации схемы.

\section{2. Кластерное состояние в непрерывных переменных}

В этом разделе кратко опишем процедуру генерации полевого линейного кластерного состояния, состоящего из четырех узлов в непрерывных переменных (рис. 1, $a$ ).

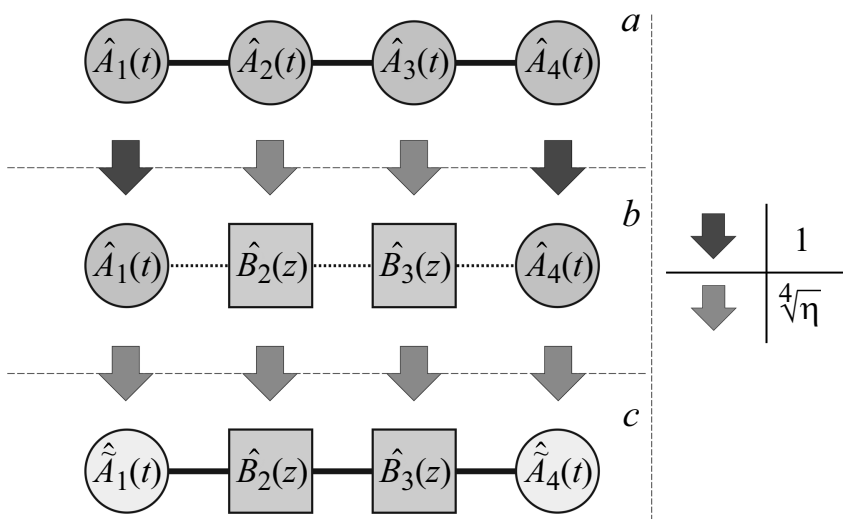

Рис. 1. Схема построения линейного четырехузлового гибридного кластерного состояния. Формирование полевого кластера заданной конфигурации (рис. $1, a$ ). Запись квантового состояния двух центральных узлов на ячейки квантовой памяти (рис. $1, b)$. Коррекция асимметрии (рис. $1, c)$.

Более детальное описание этой процедуры можно найти, например, в работе [10].

В качестве ресурса для генерации линейного кластерного состояния может быть выбран сжатый свет, излучаемый, например, оптическим параметрическим генератором (ОРО) [12] или субпуассоновым лазером с захватом фазы [13]. Поскольку далее мы хотим использовать процедуру квантовой памяти для состояния света, то необходимым требованием является работа источников света в импульсном режиме, с формированием определенной огибающей импульса. Поля на выходе четырех независимых источников света будем рассматривать в виде плоских квазимонохроматических волн, распространяющихся под малыми углами к оси $z$ (этой малостью здесь и далее мы будем пренебрегать):

$\hat{E}_{i}(\mathbf{r}, t)=i \sqrt{\frac{\hbar \omega_{0}}{2 \varepsilon_{0} c}} \hat{a}_{i}(t) e^{-i \omega_{0} t+i k z}+$ h.c., $\quad(i=1,2,3,4)$,

где нормированные амплитуды $\hat{a}_{i}(t)$ подчиняются стандартным коммутационным соотношениям для полей в свободном пространстве:

$$
\left[\hat{a}_{i}(t), \hat{a}_{j}^{\dagger}\left(t^{\prime}\right)\right]=\delta_{i j} \delta\left(t-t^{\prime}\right), \quad(i, j=1,2,3,4) .
$$

Для удобства будем считать, что источники являются одномодовыми с временным профилем моды излучения $L(t)$ и длительностью импульса $T_{W}$ (условие нормировки $\left.\int_{0}^{T_{W}} L(t) L(t) d t=1\right)$. Таким образом, нормированные амплитуды $\hat{a}_{i}(t)$ можно разложить по полному набору мод, выделив моду генерации и сгруппировав все вакуумные моды:

$$
\hat{a}_{i}(t)=L(t) \hat{e}_{i}+\mathrm{vac}, \quad(i=1,2,3,4),
$$

где $\hat{e}_{i}$ - оператор уничтожения фотонов в сжатой моде $i$-го источника, $\left(\left\langle\hat{e}_{i}^{\dagger} \hat{e}_{i}\right\rangle-\right.$ средний поток фотонов 
в $i$-й моде), vac - вакуумный вклад от всех остальных временных мод. Поскольку источники являются независимыми, операторы $\hat{e}_{i}$ подчиняются следующим коммутационным соотношениям:

$$
\left[\hat{e}_{i}, \hat{e}_{j}^{\dagger}\right]=\delta_{i j}, \quad(i, j=1,2,3,4) .
$$

Для определенности будем считать, что по своим характеристикам источники идентичны и излучают свет, сжатый по $\hat{y}$-квадратуре $\left(\hat{e}_{i}=\hat{x}_{i}+i \hat{y}_{i}\right)$ :

$$
\left\langle\left(\Delta \hat{y}_{i}\right)^{2}\right\rangle=\frac{e^{-2 r}}{4}, \quad(i=1,2,3, \ldots n),
$$

где $r$ - коэффициент сжатия.

Прежде всего сформируем на основе введенных операторов требуемое кластерное состояние. Здесь в качестве примера рассмотрим преобразования над четырехузловым линейным кластером, поскольку это минимальное по числу узлов и связей кластерное состояние, позволяющее реализовать управляемый квантовый вентиль. Согласно определению [14], каждому кластерному состоянию можно сопоставить некоторый определенный ненаправленный граф, который однозначно характеризуется матрицей смежности $S$. Для линейного кластерного состояния, состоящего из четырех узлов, данная матрица имеет следующий вид:

$$
S=\left(\begin{array}{llll}
0 & 1 & 0 & 0 \\
1 & 0 & 1 & 0 \\
0 & 1 & 0 & 1 \\
0 & 0 & 1 & 0
\end{array}\right) .
$$

Матрица смежности $S$ определяет преобразование Боголюбова $U$, с помощью которого формируется кластерное состояние, с точностью до произвольной ортогональной матрицы $Q\left(Q Q^{T}=Q^{T} Q=I\right)$ :

$$
U=(I+i S)\left(I+S^{2}\right)^{-1 / 2} Q .
$$

В результате преобразования Боголюбова $U$, осуществляемого с помощью элементов линейной оптики [15], из исходного набора нормированных амплитуд $\left\{\hat{a}_{i}(t)\right\}_{i=1}^{4}$ получится новый набор амплитуд $\left\{\hat{A}_{i}(t)\right\}_{i=1}^{4}$, которые будут являться узлами кластерного состояния:

$$
\hat{A}_{i}(t)=\sum_{j=1}^{4} u_{i j} \hat{a}_{j}(t), \quad(i=1,2,3,4),
$$

где $u_{i j}$ - элементы матрицы преобразования $U$. Поскольку преобразование Боголюбова является унитарным, оно сохранит вид коммутационных соотношений, T. e.

$$
\left[\hat{A}_{i}(t), \hat{A}_{j}^{\dagger}\left(t^{\prime}\right)\right]=\delta_{i j} \delta\left(t-t^{\prime}\right), \quad(i, j=1,2,3,4) .
$$

Оценку степени перепутанности сформированного состояния удобно производить на языке операторов нуллифайеров. Согласно [14], нуллифайеры $\hat{N}_{i}$ формируются из квадратур узлов кластерного состояния
$\hat{A}_{j}(t)=L(t)\left(\hat{X}_{j}+\hat{Y}_{j}\right)+$ vac следующим образом:

$$
\hat{N}_{i}=\hat{Y}_{i}-\sum_{j=1}^{4} s_{i j} \hat{X}_{j}, \quad(i=1,2,3,4),
$$

где $s_{i j}$ - элемент матрицы смежности $S$, определяющий силу связи между $i$-м и $j$-м узлами. Мы рассматриваем здесь так называемый невзвешенный граф, когда элементы матрицы смежности могут принимать значения 0 или 1.

Применим описанную выше процедуру к определенным нами источникам сжатого света, выбрав $Q$ в преобразовании Боголюбова (7) таким образом, чтобы минимизировать набор оптических элементов, осуществляющих преобразование. Это требование приводит к упрощению явного вида самого преобразования Боголюбова:

$$
U=\left(\begin{array}{cccc}
\frac{1}{\sqrt{2}} & \frac{1}{\sqrt{10}} & -\frac{i \sqrt{2}}{\sqrt{5}} & 0 \\
\frac{i}{\sqrt{2}} & -\frac{i}{\sqrt{10}} & -\frac{\sqrt{2}}{\sqrt{5}} & 0 \\
0 & -\frac{\sqrt{2}}{\sqrt{5}} & -\frac{i}{\sqrt{10}} & -\frac{i}{\sqrt{2}} \\
0 & -\frac{i \sqrt{2}}{\sqrt{5}} & \frac{1}{\sqrt{10}} & -\frac{1}{\sqrt{2}}
\end{array}\right) .
$$

Набор нуллифайеров для рассматриваемого линейного кластерного состояния примет вид

$$
\begin{gathered}
\hat{N}_{1}=\sqrt{2} \hat{y}_{1}, \\
\hat{N}_{2}=-\hat{y}_{4} / \sqrt{2}-\sqrt{5 / 2} \hat{y}_{3}, \\
\hat{N}_{3}=\hat{y}_{1} / \sqrt{2}-\sqrt{5 / 2} \hat{y}_{2}, \\
\hat{N}_{4}=-\sqrt{2} \hat{y}_{4} .
\end{gathered}
$$

Получившиеся нуллифайеры $\left\{\hat{N}_{i}(t)\right\}_{i=1}^{4}$ зависят только от сжатых квадратур исходных осцилляторов. Это значит, что их среднеквадратичные флуктуации при гомодинном детектировании света с временным профилем локального осциллятора $L(t)$ будут равны соответственно:

$$
\begin{gathered}
\left\langle\left(\Delta \hat{N}_{1}\right)^{2}\right\rangle=\left\langle\left(\Delta \hat{N}_{4}\right)^{2}\right\rangle=\frac{e^{-2 r}}{2}, \\
\left\langle\left(\Delta \hat{N}_{2}\right)^{2}\right\rangle=\left\langle\left(\Delta \hat{N}_{3}\right)^{2}\right\rangle=\frac{3 e^{-2 r}}{4},
\end{gathered}
$$

т. е. будут стремиться к нулю при $r \rightarrow \infty$. Таким образом, согласно [14], полученное состояние действительно является кластерным.

\section{3. Формирование гибридного кластерного состояния}

Световое кластерное состояние является оптимальным инструментом для ввода и вывода информации в 
процессе квантовых вычислений. Однако сами вычисления могут занимать значительное время. Например, проведение вычислений может быть обусловлено какимлибо событием (результатом другого вычисления) или же разнесено в пространстве. С этих позиций сохранение узлов кластера в виде световых полей требует слишком большой петли задержки, и потому затруднительно.

Роль такой линии задержки могут выполнять ячейки квантовой памяти - ансамбли атомов, на коллективную когерентность которых можно „отпечатать“ квантовое состояние светового импульса. В этом разделе мы кратко обсудим процедуру формирования гибридного кластерного состояния, когда часть узлов кластера остаются световыми полями $\left\{\hat{A}_{i}(t)\right\}$ (и могут использоваться для ввода и вывода информации), а другая часть узлов преобразуется в коллективные спиновые волны $\left\{\hat{\boldsymbol{B}}_{j}(z)\right\}$ атомных ансамблей (рис. $1, b)$.

Для переноса квантового состояния света на ансамбль атомов будем иметь в виду один из протоколов многомодовой квантовой памяти, описанный, например, в работах [16-18]. Напомним, что понятие квантовой памяти обычно подразумевает три последовательных этапа: запись, хранение и считывание. В процессе записи на ансамбль атомов подается сигнальное квантовое поле, статистику которого хотят сохранить, в сопровождении управляющего классического поля. Вследствие взаимодействия этих полей с ансамблем атомов формируется спиновая волна, на которую отпечатываются квантовостатистические свойства сигнального поля. Процесс хранения организуют таким образом, чтобы влиянием диссипативных процессов на этом этапе можно было полностью пренебречь, и сформированная спиновая когерентность оставалась неизменной вплоть до момента считывания. Наконец, в процессе считывания импульс управляющего поля, поданный на ячейку памяти, восстанавливает исходное сигнальное поле вместе со всеми его квантово-статистическими свойствами.

Для переноса квантового состояния света на атомный ансамбль могут быть задействованы разные механизмы взаимодействия света и вещества. Для узкополосного света удобным и эффективным является протокол памяти, базирующийся на эффекте электромагнитной индуцированной прозрачности [19-24]. Для хранения более коротких импульсов света используют протоколы рамановского взаимодействия [25-29], AFC-протоколы [3034], протокол быстрой резонансной памяти [18], QNDпротокол [35-38]. Все перечисленные выше схемы обладают общим свойством: они описываются линейным гамильтонианом взаимодействия операторов медленно меняющейся амплитуды поля и коллективных бозонных операторов спиновых волн среды. Это позволяет рассматривать задачу в виде интегрального преобразования, связывающего оператор поля на входе ячейки памяти с коллективным оператором спиновой волны. Ядра интегральных преобразований оказываются различными для разных протоколов памяти. Для конкретности далее будем говорить о протоколе рамановской памяти [29].
Отдельного внимания требует вопрос записи сигнала с конкретным требуемым временным профилем. Разработано несколько методов, обеспечивающих эффективное взаимодействие атомного ансамбля с выделенной временной модой сигнального поля. Все они основаны на выборе подходящего профиля управляющего поля. В работе [39] авторы предлагают искать такой профиль методом множителей Лагранжа. В работе [40] используется итерационная процедура поиска формы управляющего сигнала, факторизующего ядро интегрального преобразования. Для дальнейшего построения здесь нам удобнее использовать этот метод.

Процесс записи $i$-го узла $\hat{A}_{i}(t)$ светового кластерного состояния можно описать с помощью следующего интегрального преобразования:

$$
\hat{B}_{i}(z)=\int_{0}^{T_{W}} G_{a b}(t, z) \hat{A}_{i}(t) d t+\mathrm{vac},
$$

где $T_{W}$ - время взаимодействия, соответствующее длительности записываемого импульса, $G_{a b}(t, z)$ - ядро преобразования сигнального поля на этапе записи (функция Грина). Вклад vас формируется всеми модами поля кроме сигнальной, находящимися в вакуумном состоянии. Ядро $G_{a b}(t, z)$ зависит от профиля управляющего поля, что позволяет подобрать форму управляющего поля таким образом [40], чтобы его явный вид мог быть выражен в виде произведения временного профиля $L(t)$ поля, которое хотим записать в ячейку, на пространственный профиль $g(z)$, образовавшейся в ячейке спиновой волны:

$$
G_{a b}(t, z)=L(t) g(z)
$$

Таким образом, к концу процесса записи $i$-го узла кластерного состояния внутри ячейки памяти образуется спиновая волна $\hat{B}_{i}(z)$ такая, что:

$\hat{B}_{i}(z)=\int_{0}^{T_{W}} L(t) g(z) \hat{A}_{i}(t) d t+v a c=g(z)\left(\hat{X}_{i}+i \hat{Y}_{i}\right)+$ vac.

Последнее равенство использует условие нормировки огибающей $L(t)$. Из (20) видно, что операторы квадратур $\hat{X}_{i}$ и $\hat{Y}_{i}$ спиновой волны $\hat{B}_{i}(z)$ совпадают с операторами квадратур записанного узла светового кластера $\hat{A}_{i}(t)=L(t)\left(\hat{X}_{i}+\hat{Y}_{i}\right)+$ vac. Благодаря выбранному виду ядра (19) ячейки памяти взаимодействуют только с модой поля с временным профилем $L(t)$, другие пространственные моды не возбуждаются, они остаются в вакуумном состоянии. Указанная процедура записи узла кластерного состояния в ячейку памяти позволяет сформировать гибридное кластерное состояние. 


\section{4. Роль симметрии в формировании гибридного кластерного состояния}

Рассмотренная выше ситуация соответствует идеальной процедуре квантовой памяти, работающей со $100 \%$ (единичной) эффективностью. На практике эффективность памяти может оказаться меньше. Когда на ячейку памяти записывается все квантовое состояние системы, потеря эффективности работает как ,передающий коэффициент“; линии связи, и квантовая статистика сохраняется в меру величины этого передающего коэффициента. Однако ситуация отличается кардинально, если процедуре памяти подвергается только часть подсистем сложной квантовой системы, обладающей перепутыванием. Этот вопрос был исследован в работах [41,42]. Авторы [41] рассматривали в деталях следующий мысленный эксперимент: два импульса сжатого света перепутывали на светоделительной пластинке, после чего один из импульсов сохранялся в ячейке квантовой памяти, а другой не участвовал в процессе записи-восстановления, и распространялся неизменным в линии задержки. Было показано, что такая асимметричная схема распространения сохраняет перепутывание в системе только если эффективность квантовой памяти близка к единичной. В противном случае оказывается, что дефект перепутывания двух подсистем будет определяться не только сжатыми, но и растянутыми квадратурами, и соответственно перепутывание легко разрушается.

Применяя те же рассуждения к рассматриваемой нами системе, нетрудно заметить, что запись лишь части узлов кластера создает асимметричные условия для некоторых связей. Рассмотрим данный вопрос на конкретном примере гибридного кластерного состояния.

Запишем узлы $\hat{A}_{2}(t)$ и $\hat{A}_{3}(t)$ линейного кластера в две ячейки рамановской квантовой памяти (рис. $1, b$ ). Если считать, что квантовая память работает с единичной эффективностью, результирующее состояние можно представить набором узлов:

$$
\begin{aligned}
& \hat{A}_{1}(t)=L(t)\left(\hat{X}_{1}+i \hat{Y}_{1}\right)+\mathrm{vac}, \\
& \hat{B}_{2}(z)=g(z)\left(\hat{X}_{2}+i \hat{Y}_{2}\right)+\mathrm{vac}, \\
& \hat{B}_{3}(z)=g(z)\left(\hat{X}_{3}+i \hat{Y}_{3}\right)+\mathrm{vac}, \\
& \hat{A}_{4}(t)=L(t)\left(\hat{X}_{4}+i \hat{Y}_{4}\right)+\mathrm{vac} .
\end{aligned}
$$

Если мы составим для них нуллифайеры согласно (10), то получим полное совпадение с системой нуллифайеров (12)-(15). Поскольку условия (16) и (17), были выполнены для нуллифайеров полевого кластерного состояния, они будут также выполнены и для узлов гибридного кластерного состояния. Это подтверждается тем, что, согласно [40], процесс записи оказывается обратимым и при считывании из ячейки памяти узлов $\hat{B}_{2}(z)$ и $\hat{B}_{3}(z)$, мы восстановим исходное кластерное состояния со всеми его квантово-статистическими особенностями. Таким образом, можно утверждать, что при идеальной записи узлов кластерного состояния на ячейки памяти, сама кластерная структура остается без изменений, и связь между узлами сохраняется.

Совсем иная ситуация возникает при неидеальной записи. Тогда две из трех линий связи оказываются в асимметричных условиях. Если при этом мы попытаемся оценить перепутывание по тому же рецепту, что и прежде, то увидим, что в нуллифайеры дадут вклад не только сжатые квадратурные компоненты $\left\{y_{i}\right\}$, но и растянутые квадратуры $\left\{x_{i}\right\}$ исходных состояний, т.е. сформированные таким образом операторы перестанут быть нуллифайерами. В самом деле, будем описывать результирующее состояние системы с учетом эффективности квантовой памяти $\eta$ через операторы узлов:

$$
\begin{gathered}
\hat{A}_{1}(t)=L(t)\left(\hat{X}_{1}+i \hat{Y}_{1}\right)+\mathrm{vac}, \\
\hat{B}_{2}(z)=g(z)\left(\sqrt[4]{\eta}\left(\hat{X}_{2}+i \hat{Y}_{2}\right)\right. \\
\left.+\sqrt{1-\sqrt{\eta}}\left(\hat{x}_{v 2}+i \hat{y}_{v 2}\right)\right)+\mathrm{vac}, \\
\hat{B}_{3}(z)=g(z)\left(\sqrt[4]{\eta}\left(\hat{X}_{3}+i \hat{Y}_{3}\right)\right. \\
\left.+\sqrt{1-\sqrt{\eta}}\left(\hat{x}_{v 3}+i \hat{y}_{v 3}\right)\right)+\mathrm{vac} \\
\hat{A}_{4}(t)=L(t)\left(\hat{X}_{4}+i \hat{Y}_{4}\right)+\mathrm{vac} .
\end{gathered}
$$

Здесь операторы $\hat{x}_{v 2}, \hat{y}_{v 2}, \hat{x}_{v 3}, \hat{y}_{v 3}$ соответствуют вакуумному шуму, который добавляется в интересующую нас пространственную моду с профилем $g(z)$. Эти операторы восстанавливают исходные коммутационные соотношения спиновых волн $\hat{B}_{2}$ и $\hat{B}_{3}$ и описываются своими дисперсиями:

$$
\left\langle\left(\Delta \hat{x}_{v i}\right)^{2}\right\rangle=\left\langle\left(\Delta \hat{y}_{v i}\right)^{2}\right\rangle=\frac{1}{4}, \quad i=2,3 .
$$

Индекс $v$ указывает на вакуумный характер добавляемых шумов. Можно сказать, что квантовая память работает для записываемой моды поля как светоделитель с амплитудным коэффициентом пропускания $\sqrt[4]{\eta}$ и соответствующим коэффициентом отражения $\sqrt{1-\sqrt{\eta}}$.

Пользуясь тем же рецептом построения нуллифайеров, получим:

$$
\begin{gathered}
\hat{N}_{1}=\frac{\hat{y}_{2}(1-\sqrt[4]{\eta})+\sqrt{5} \hat{y}_{1}(\sqrt[4]{\eta}+1)}{\sqrt{10}} \\
-\frac{2 \hat{x}_{3}(1-\sqrt[4]{\eta})}{\sqrt{10}}-\hat{x}_{v 2} \sqrt{1-\sqrt{\eta}}, \\
\hat{N}_{2}=-\frac{\hat{y}_{3}(3 \sqrt[4]{\eta}+2)+\sqrt{5} \hat{y}_{4} \sqrt[4]{\eta}}{\sqrt{10}} \\
-\frac{\left(\sqrt{5} \hat{x}_{1}+\hat{x}_{2}\right)(1-\sqrt[4]{\eta})}{\sqrt{10}}+\left(\hat{x}_{v 3}-\hat{y}_{v 2}\right) \sqrt{1-\sqrt{\eta}},
\end{gathered}
$$




$$
\begin{gathered}
\hat{N}_{3}=-\frac{\hat{y}_{2}(3 \sqrt[4]{\eta}+2)-\sqrt{5} \hat{y}_{1} \sqrt[4]{\eta}}{\sqrt{10}} \\
\left.+\frac{\left(\sqrt{5} \hat{x}_{4}-\hat{x}_{3}\right)(1-\sqrt[4]{\eta})}{\sqrt{10}}-\left(\hat{x}_{v 2}-\hat{y}_{v 3}\right) \sqrt{1-\sqrt{\eta}}\right), \\
\hat{N}_{4}=\frac{\hat{y}_{3}(1-\sqrt[4]{\eta})-\sqrt{5} \hat{y}_{4}(\sqrt[4]{\eta}+1)}{\sqrt{10}} \\
-\frac{2 \hat{x}_{2}(1-\sqrt[4]{\eta})}{\sqrt{10}}-\hat{x}_{v 3} \sqrt{1-\sqrt{\eta}}
\end{gathered}
$$

Наличие асимметрии приводит к тому, что операторы $\left\{\hat{N}_{i}\right\}_{i=1}^{4}$ теперь зависят не только от сжатых квадратур (первые слагаемые в выражениях (30)-(33) и от квадратур добавочных вакуумных шумов (третьи слагаемые), но и от растянутых квадратур (вторые слагаемые). Очевидно, что эти операторы не стремятся к нулю при $r \rightarrow \infty$, т.е. не удовлетворяют определению нуллифайеров.

Исправить ситуацию можно, симметризовав потери. Введем дополнительные потери в каналах $\hat{A}_{1}(t)$ и $\hat{A}_{4}(t)$, такие потери можно условно смоделировать зеркалами с коэффициентом пропускания $\sqrt[4]{\eta}$ и коэффициентом отражения $\sqrt{1-\sqrt{\eta}}$ по амплитуде (рис. $1, c)$ :

$$
\begin{aligned}
\hat{\tilde{A}}_{1}(t)= & L(t)\left(\sqrt[4]{\eta}\left(\hat{X}_{1}+i \hat{Y}_{1}\right)+\sqrt{1-\sqrt{\eta}}\left(\hat{x}_{v 1}+i \hat{y}_{v 1}\right)\right) \\
& +\operatorname{vac}=L(t)\left(\hat{\tilde{X}}_{1}+i \hat{\tilde{Y}}_{1}\right)+\mathrm{vac}, \\
\hat{\tilde{A}}_{4}(t)= & L(t)\left(\sqrt[4]{\eta}\left(\hat{X}_{4}+i \hat{Y}_{4}\right)+\sqrt{1-\sqrt{\eta}}\left(\hat{x}_{v 4}+i \hat{y}_{v 4}\right)\right) \\
& +\operatorname{vac}=L(t)\left(\hat{\tilde{X}}_{4}+i \hat{\tilde{Y}}_{4}\right)+\mathrm{vac},
\end{aligned}
$$

где операторы $\hat{x}_{v 1}, \hat{y}_{v 1}, \hat{x}_{v 4}, \hat{y}_{v 4}$ соответствуют вакуумному шуму, который добавляется во временную моду с профилем $L(t)$, и определены аналогично (29).

В этом случае ситуация, безусловно, исправится, и нуллифайеры примут вид

$$
\begin{gathered}
\hat{N}_{1}=\sqrt{2} \hat{y}_{1} \sqrt[4]{\eta}+\left(\hat{y}_{v 1}-\hat{x}_{v 2}\right) \sqrt{1-\sqrt{\eta}} \\
\hat{N}_{2}=-\left(\sqrt{5} \hat{y}_{3}+\hat{y}_{4}\right) \sqrt[4]{\eta} / \sqrt{2}+\left(\hat{y}_{v 2}-\hat{x}_{v 1}-\hat{x}_{v 3}\right) \sqrt{1-\sqrt{\eta}} \\
\hat{N}_{3}=-\left(\sqrt{5} \hat{y}_{2}-\hat{y}_{1}\right) \sqrt[4]{\eta} / \sqrt{2} \\
+\left(\hat{y}_{v 3}-\hat{x}_{v 2}-\hat{x}_{v 4}\right) \sqrt{1-\sqrt{\eta}} \\
\hat{N}_{4}=-\sqrt{2} \hat{y}_{4} \sqrt[4]{\eta}+\left(\hat{y}_{v 4}-\hat{x}_{v 3}\right) \sqrt{1-\sqrt{\eta}}
\end{gathered}
$$

Эти операторы, хотя и „подпорчены“ вакуумными слагаемыми, тем не менее уже не содержат растянутых квадратур.

Интуитивно кажется, что введение дополнительных потерь в квантовых каналах является нежелательной процедурой с точки зрения информационных свойств каналов. Однако, как мы покажем в следующем разделе, оно позволяет проводить квантовые вычисления и почти не влияет на их результат (в силу малости величины $\sqrt{1-\sqrt{\eta}})$, в то время как без подобной симметризации протокол вычислений разрушается.

\section{5. Реализация логической операции $C Z$ при помощи гибридного кластерного состояния}

Рассмотрим применение гибридных кластерных состояний для реализации на их основе однонаправленных квантовых логических операций. Напомним, что однонаправленные логические операции осуществляются путем последовательных проекционных измерений узлов кластерного состояния, так что с каждым измерением размерность кластерного состояния поэтапно уменьшается и остается лишь один или несколько узлов, которые содержат результат выполненной логической операции. Нас будет интересовать ситуация, в которой результат выполненной логической операции окажется на атомных узлах кластерного состояния, записанных в ячейках квантовой памяти.

В качестве примера рассмотрим двухмодовую логическую операцию $C Z$ [43]. Отметим, что принципиально результат не будет зависеть от типа логического вентиля. Будем следовать логике проведения преобразований, представленной в работе [11], выполняя вычисления с помощью кластера, состоящего из минимального количества требуемых для нашего вычисления узлов (четырехузлового), обсуждавшегося в предыдущем разделе. Важно также отметить, что само понятие управляемых вентилей в непрерывных переменных несколько отличается от аналогичных преобразований в дискретных переменных. Двухмодовая операция применяется к двум исходно независимым модам, которые будем описывать операторами Гейзенберга для медленных амплитуд:

$$
\begin{aligned}
& \hat{A}_{\text {in } 1}(t)=L(t)\left(\hat{X}_{\text {in } 1}+i \hat{Y}_{\text {in } 1}\right)+\text { vac }, \\
& \hat{A}_{\text {in } 2}(t)=L(t)\left(\hat{X}_{\text {in } 2}+i \hat{Y}_{\text {in } 2}\right)+\text { vac. }
\end{aligned}
$$

Для простоты мы положили, что каждая из этих мод обладает тем же временным профилем $L(t)$, что и узлы светового кластерного состояния. Отметим, что в случае разных временных профилей мы не сможем гарантировать эффективное взаимодействие световых полей на элементах линейной оптики, с помощью которых выполняются все последующие преобразования [44].

Результатом операции $C Z$ называют преобразование этих мод по следующему закону:

$$
\begin{aligned}
& \hat{A}_{\text {out } 1}(t)=L(t)\left(\hat{X}_{\text {out } 1}+i \hat{Y}_{\text {out } 1}\right)=L(t)\left(\hat{X}_{\text {in } 1}+i\left(\hat{Y}_{\text {in } 1}+\hat{X}_{\text {in } 2)}\right),\right. \\
& \hat{A}_{\text {out } 2}(t)=L(t)\left(\hat{X}_{\text {out } 2}+i \hat{Y}_{\text {out } 2}\right)=L(t)\left(\hat{X}_{\text {in } 2}+i\left(\hat{Y}_{\text {in } 2}+\hat{X}_{\text {in } 1}\right)\right) .
\end{aligned}
$$




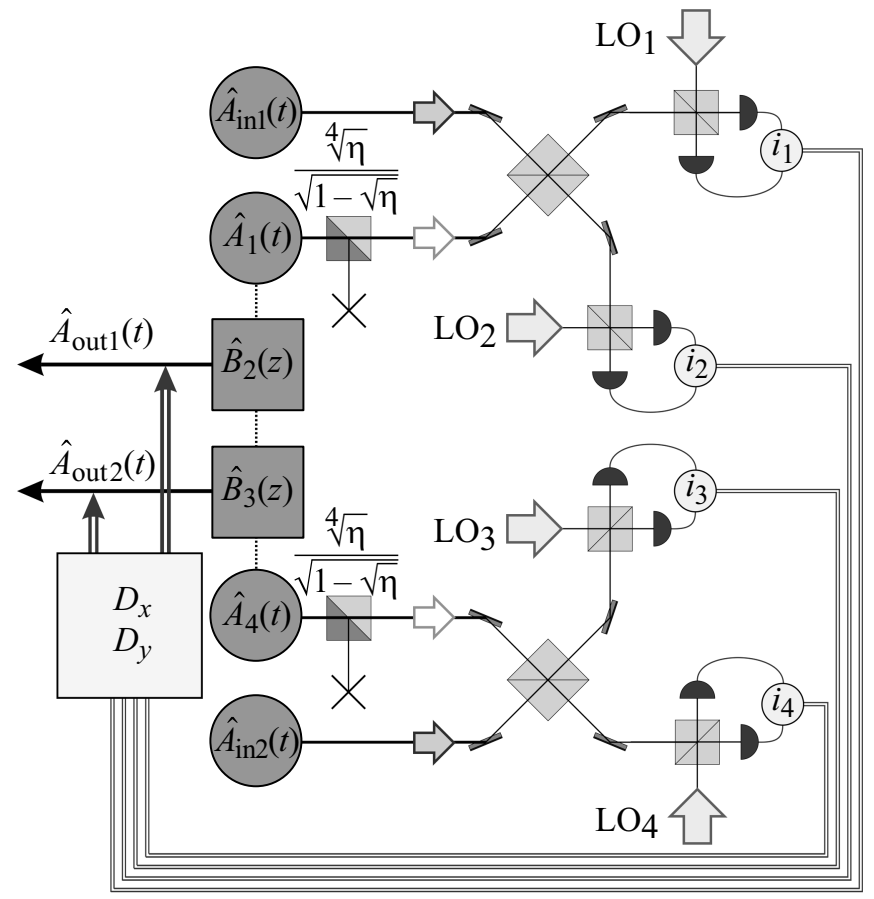

Рис. 2. Операция $C Z$ на гибридном 4-модовом линейном кластере. К крайним (полевым) узлам кластерного состояния $\tilde{\tilde{A}}_{1}(t)$ и $\tilde{\tilde{A}}_{4}(t)$ при помощи светоделителей примешиваются входные моды $\hat{A}_{\text {in } 1}(t)$ и $\hat{A}_{\text {in } 2}(t)$. Далее поля со всех выходов светоделителей детектируются методом гомодинного детектирования (профиль локальных осцилляторов $\mathrm{LO}_{k}, k=1,2,3,4$, совпадает с профилем световых мод кластера). Конкретный вид вентиля осуществляется именно на этом этапе путем выбора определенного набора значений для фаз локальных осцилляторов (например, вентиль $C Z$ реализуется при выборе фазовых значений $\left.\theta_{1}=-\pi / 4, \theta_{2}=-3 \pi / 4, \theta_{3}=3 \pi / 4 /, \theta_{4}=\pi / 4\right)$. В результате центральные (материальные) узлы кластерного состояния $\hat{B}_{2}(z)$ и $\hat{B}_{3}(z)$ с точностью до сдвигов содержат результат произведенных вычислений. Результат может храниться требуемое время (ограниченное временем распада квантовой памяти). При считывании необходимо включить в схему операции компенсации сдвига $D x$ и $D y$.

В результате такого преобразования $\hat{X}$-квадратуры (управляющие) не изменились, а $\hat{Y}$-квадратуры (управляемые) изменились в зависимости от $\hat{X}$-квадратур. Преобразование $C Z$ удобно описывать в матричной форме:

$$
C Z=\left(\begin{array}{llll}
1 & 0 & 0 & 0 \\
0 & 1 & 0 & 0 \\
0 & 1 & 1 & 0 \\
1 & 0 & 0 & 1
\end{array}\right),
$$

действующей на вектор-столбец, компонентами которого являются квадратуры входных состояний $\left(\hat{X}_{\text {in } 1}(t), \hat{X}_{\text {in } 2}(t), \hat{Y}_{\text {in } 1}(t), \hat{Y}_{\text {in } 2}(t)\right)^{T}$.

Входные моды (40) и (41) примешаем к крайним (полевым) узлам гибридного кластерного состояния (первому и четвертому) посредством симметричных светоделителей (рис. 2):

$$
\begin{aligned}
& \frac{1}{\sqrt{2}}\left(\hat{A}_{\mathrm{in} 1}(t)+\hat{\tilde{\tilde{A}}}_{1}(t)\right)=\frac{L(t)}{\sqrt{2}}\left(\hat{X}_{\mathrm{in} 1}+\hat{\tilde{X}}_{1}+i\left(\hat{Y}_{\mathrm{in} 1}+\hat{\tilde{Y}}_{1}\right)\right)+\mathrm{vac}, \\
& \frac{1}{\sqrt{2}}\left(\hat{A}_{\mathrm{in} 1}(t)-\hat{\tilde{A}}_{1}(t)\right)=\frac{L(t)}{\sqrt{2}}\left(\hat{X}_{\mathrm{in} 1}-\hat{\tilde{X}}_{1}+i\left(\hat{Y}_{\mathrm{in} 1}-\hat{\tilde{Y}}_{1}\right)\right)+\mathrm{vac}, \\
& \frac{1}{\sqrt{2}}\left(\hat{A}_{\mathrm{in} 2}(t)+\hat{\tilde{A}}_{4}(t)\right)=\frac{L(t)}{\sqrt{2}}\left(\hat{X}_{\mathrm{in} 2}+\hat{\tilde{X}}_{4}+i\left(\hat{Y}_{\mathrm{in} 2}+\hat{\tilde{Y}}_{4}\right)\right)+\mathrm{vac}, \\
& \frac{1}{\sqrt{2}}\left(\hat{A}_{\mathrm{in} 2}(t)-\hat{\tilde{A}}_{4}(t)\right)=\frac{L(t)}{\sqrt{2}}\left(\hat{X}_{\mathrm{in} 2}-\hat{\tilde{X}}_{4}+i\left(\hat{Y}_{\mathrm{in} 2}-\hat{\tilde{Y}}_{4}\right)\right)+\mathrm{vac} .
\end{aligned}
$$

Далее эти четыре поля детектируются методом гомодинного детектирования. Все четыре гомодина (локальные осцилляторы) $\beta(t) e^{i \theta_{k}}$ имеют одинаковый временной профиль $\beta(t)=\beta_{0} L(t)$ (что позволит нивелировать вклад от вакуумных слагаемых), но разные фазы $\left\{\theta_{k}\right\}_{k=1}^{4}$. Выбор именно этих фазовых множителей позволяет контролировать какая логическая операция будет произведена. На этом этапе вычислений мы оставляем фазы гомодинов свободными параметрами расчета.

Результат измерения определяется операторами фототоков:

$$
\begin{aligned}
& \hat{i_{1}}=\beta_{0} \sqrt{2}\left[\cos \left[\theta_{1}\right]\left(\hat{X}_{\text {in } 1}+\hat{\tilde{X}}_{1}\right)+\sin \left[\theta_{1}\right]\left(\hat{Y}_{\text {in } 1}+\hat{\tilde{Y}}_{1}\right)\right], \\
& \hat{i}_{2}=\beta_{0} \sqrt{2}\left[\cos \left[\theta_{2}\right]\left(\hat{X}_{\text {in1 }}-\hat{\tilde{X}}_{1}\right)+\sin \left[\theta_{2}\right]\left(\hat{Y}_{\text {in } 1}-\tilde{\tilde{Y}}_{1}\right)\right], \\
& \hat{i}_{3}=\beta_{0} \sqrt{2}\left[\cos \left[\theta_{3}\right]\left(\hat{X}_{\text {in2 }}+\hat{\tilde{X}}_{4}\right)+\sin \left[\theta_{3}\right]\left(\hat{Y}_{\text {in } 2}+\hat{\tilde{Y}}_{4}\right)\right], \\
& \hat{i}_{4}=\beta_{0} \sqrt{2}\left[\cos \left[\theta_{4}\right]\left(\hat{X}_{\text {in2 }}-\hat{\tilde{X}}_{4}\right)+\sin \left[\theta_{4}\right]\left(\hat{Y}_{\text {in } 2}-\hat{\tilde{Y}}_{4}\right)\right] .
\end{aligned}
$$

Здесь $\left\{\hat{i}_{k}\right\}$ - интегрированные по времени разностные токи. Отметим, что в силу перепутанности системы эти измерения меняют состояния материальных узлов кластера.

Воспользуемся связью между квадратурами кластерного состояния и квадратурами источников сжатого света (8). Решим систему (49)-(52), выражая растянутые квадратуры $\left\{\hat{x}_{i}\right\}$ как функции от квадратур входных полей $\left\{\hat{X}_{\text {in } 1}, \hat{X}_{\text {in2 } 2}, \hat{Y}_{\text {in } 1}, \hat{Y}_{\text {in } 2}\right\}$, интегрированных токов $\left\{\hat{i}_{k}\right\}$, сжатых квадратур исходных осцилляторов $\left\{\hat{y}_{k}\right\}$ и эффективности $\eta$. Подставим полученные решения в выражения (26), (27) для амплитуд $\hat{B}_{2}(z)$ и $\hat{B}_{3}(z)$ с учетом связи (8). Таким образом мы найдем квадратуры материальных осцилляторов кластерного состояния, которые являются для данного преобразования выходными модами: $\hat{B}_{2}(z) \rightarrow \hat{B}_{\text {out } 1}(z)=g(z)\left(\hat{X}_{\text {out } 1}+i \hat{Y}_{\text {out } 1}\right)+$ vac и $\hat{B}_{3}(z) \rightarrow \hat{B}_{\text {out } 2}(z)=g(z)\left(\hat{X}_{\text {out } 2}+i \hat{Y}_{\text {out } 2}\right)+$ vac.

Введем обозначения

$$
\begin{gathered}
\hat{\mathbf{E}}_{\text {in }} \equiv\left(\hat{X}_{\text {in } 1}, \hat{X}_{\text {in2 }}, \hat{Y}_{\text {in } 1}, \hat{Y}_{\text {in } 2}\right)^{T}, \\
\hat{\mathbf{E}}_{\text {out }} \equiv\left(\hat{X}_{\text {out } 1}, \hat{X}_{\text {out } 2}, \hat{Y}_{\text {out } 1}, \hat{Y}_{\text {out } 2}\right)^{T}, \\
\hat{\mathbf{y}} \equiv\left(\hat{y}_{1}, \hat{y}_{2}, \hat{y}_{3}, \hat{y}_{4}\right)^{T},
\end{gathered}
$$




$$
\begin{gathered}
\hat{\mathbf{i}} \equiv\left(\hat{i}_{1}, \hat{i}_{2}, \hat{i}_{3}, \hat{i}_{4}\right)^{T}, \\
\hat{\mathbf{v}} \equiv\left(\hat{x}_{\mathrm{v} 1}, \hat{x}_{\mathrm{v} 2}, \hat{x}_{\mathrm{v} 3}, \hat{x}_{\mathrm{v} 4}, \hat{y}_{\mathrm{v} 1}, \hat{y}_{\mathrm{v} 2}, \hat{y}_{\mathrm{v} 3}, \hat{y}_{\mathrm{v} 4}\right)^{T} .
\end{gathered}
$$

Тогда результат вычисления можно записать в векторной форме:

$$
\hat{\mathbf{E}}_{\text {out }}=K \hat{\mathbf{E}}_{\text {in }}+\sqrt[4]{\eta} F \hat{\mathbf{y}}+G \hat{\mathbf{i}}+\sqrt{1-\sqrt{\eta}} N \hat{\mathbf{v}},
$$

где явный вид матриц преобразований $K$ и $G$ зависит только от фаз локальных осцилляторов, выбранных при гомодинном детектировании; матрица $F$ в данном случае определена только конфигурацией кластера (т.е. матрицей связности $S$, использованной при построении полевого кластера) и не зависит ни от фаз гомодинов, ни от эффективности памяти. Явный вид всех этих матриц приведен в Приложении А.

Первое слагаемое в выражении (58) отвечает желаемому логическому преобразованию над квадратурами. Выбирая фазы локальных осцилляторов, например, равными $\theta_{1}=-\pi / 4, \theta_{2}=-3 \pi / 4, \theta_{3}=3 \pi / 4, \theta_{4}=\pi / 4$, мы можем осуществить желаемое преобразование $K=C Z$. Второе слагаемое мало, поскольку содержит только сжатые квадратуры $\hat{\mathbf{y}}$ с числовыми коэффициентами. Третье слагаемое, пропорциональное операторам фототоков, может быть скомпенсировано при дальнейшем считывании выходных сигналов из ячеек памяти. В схеме вычислений на кластерных состояниях мы измеряем фототоки, поэтому должны перейти от операторов $\hat{\mathbf{i}}$ к соответствующим измеренным $c$-числовым значениям. Тогда в третьем слагаемом правой части окажутся только классические величины, которые могут быть скомпенсировать при смещения квадратур выходного поля с использованием процедуры feed-forward [43]. Наконец, последнее слагаемое в выражении (58) оказывается малым при достаточно большой эффективности квантовой памяти $\eta$. Таким образом, полученное преобразование позволяет сохранить в ячейках квантовой памяти результат вычислений. Точность преобразования будет определяться исходным сжатием световых осцилляторов, из которых конструируется кластер, а также эффективностью используемых ячеек памяти.

Таким образом мы выполнили управляемое логическое преобразование $C Z$, результат которого можно сохранять в ячейке памяти и восстановить по требованию для следующего этапа вычислений.

\section{6. Заключение}

Мы показали как сформировать гибридное кластерное состояние на основе сжатых световых мод и ячеек квантовой памяти, а также оценили точность квантовых вычислений на таком состоянии, принимая во внимание как ошибки, связанные с конечным сжатием используемых световых полей, так и неидеальность хранения информации. Вид кластерного состояния, выбранный для анализа, определяется тем, что такое состояние обладает минимальным возможным числом узлов и связей, достаточным для выполнения как одномодовых, так и управляемых двухмодовых операций. Еще раз подчеркнем, что предложенная архитектура не требует построения больших многоузловых кластерных состояний и допускает продолжительные временные интервалы между отдельными логическими операциями.

Конечная эффективность процесса переноса состояния узлов кластера со световых осцилляторов на атомные приводит к разрушению кластерного состояния системы даже при высоких (но всеже отличных от 1) значениях эффективности. Однако если скомпенсировать возникающую асимметрию, то удается восстановить запутанность узлов. Мы показали, что внесение светоделительных пластинок позволяет восстановить „разбалансировку“; кластера, что, в свою очередь, дает возможность выполнить вычисления с высокой точностью.

Хотя идея формирования атомно-полевых кластерных состояний была предложена ранее в работе [8], нам кажется, что обсуждаемое здесь решение более „экономично“. В работе [8] авторы, используя QND-измерения, сперва формировали четырехузловое материальное кластерное состояние, отвечающее квадратному графу, а затем „привязывали“; к нему одну световую моду для управления. Выполняя те же функции, такой подход требует большего ресурса сжатых состояний, чем предложенный нами (шесть сжатых световых мод вместо четырех), а также задействует большее число атомных ансамблей (четыре вместо двух).

Мы провели вычисления, используя в качестве ресурса для построения кластера одинаково сжатые световые осцилляторы. Если не ограничиваться этим случаем, а рассмотреть моды с произвольным сжатием, то можно подобрать условия более выгодного распределения ресурса сжатия/перепутывания по узлам светового кластера, что возможно позволит избежать включения в схему дополнительных элементов потерь и уменьшит ошибки вычисления. Этот вопрос требует дальнейшего анализа.

\section{Финансирование работы}

Работа выполнена при финансовой поддержке Российского научного фонда (грант № 17-72-10171).

\section{Конфликт интересов}

Авторы заявляют, что у них нет конфликта интересов.

\section{Приложение А. Матричные коэффициенты преобразования квадратур при выполнении двухмодовой операции}

Раскроем явный вид квадратур матричных коэффициентов в выражении (58), описывающем связь входных и выходных квадратур при двухмодовой управляемой 
логической операции на линейном четырехузловом кластере:

$$
\begin{aligned}
& K= \\
& =\left(\begin{array}{cccc}
-\frac{2 \cos \left[\theta_{1}\right] \cos \left[\theta_{2}\right]}{\sin \left[\theta_{1}-\theta_{2}\right]} & 0 & -\frac{\sin \left[\theta_{1}+\theta_{2}\right]}{\sin \left[\theta_{1}-\theta_{2}\right]} & 0 \\
0 & -\frac{2 \cos \left[\theta_{3}\right] \cos \left[\theta_{4}\right]}{\sin \left[\theta_{3}-\theta_{4}\right]} & 0 & -\frac{\sin \left[\theta_{3}+\theta_{4}\right]}{\sin \left[\theta_{3}-\theta_{4}\right]} \\
\frac{\sin \left[\theta_{1}+\theta_{2}\right]}{\sin \left[\theta_{1}-\theta_{2}\right]} & -\frac{2 \cos \left[\theta_{3}\right] \cos \left[\theta_{4}\right]}{\sin \left[\theta_{3}-\theta_{4}\right]} & \frac{2 \sin \left[\theta_{1}\right] \sin \left[\theta_{2}\right]}{\sin \left[\theta_{1}-\theta_{2}\right]} & -\frac{\sin \left[\theta_{3}+\theta_{4}\right]}{\sin \left[\theta_{3}-\theta_{4}\right]} \\
-\frac{2 \cos \left[\theta_{1}\right] \cos \left[\theta_{2}\right]}{\sin \left[\theta_{1}-\theta_{2}\right]} & \frac{\sin \left[\theta_{3}+\theta_{4}\right]}{\sin \left[\theta_{3}-\theta_{4}\right]} & -\frac{\sin \left[\theta_{1}+\theta_{2}\right]}{\sin \left[\theta_{1}-\theta_{2}\right]} & \frac{2 \sin \left[\theta_{3}\right] \sin \left[\theta_{4}\right]}{\sin \left[\theta_{3}-\theta_{4}\right]}
\end{array}\right), \\
& F=\left(\begin{array}{cccc}
-\sqrt{2} & 0 & 0 & 0 \\
0 & 0 & 0 & \sqrt{2} \\
0 & 0 & -\frac{\sqrt{5}}{\sqrt{2}} & \frac{1}{\sqrt{2}} \\
-\frac{1}{\sqrt{2}} & -\frac{\sqrt{5}}{\sqrt{2}} & 0 & 0
\end{array}\right) \\
& G=\frac{\sqrt{2}}{2 \beta_{0}} \\
& \times\left(\begin{array}{cccc}
\frac{\cos \left[\theta_{2}\right]}{\sin \left[\theta_{1}-\theta_{2}\right]} & \frac{\cos \left[\theta_{1}\right]}{\sin \left[\theta_{1}-\theta_{2}\right]} & 0 & 0 \\
0 & 0 & \frac{\cos \left[\theta_{4}\right]}{\sin \left[\theta_{3}-\theta_{4}\right]} & \frac{\cos \left[\theta_{3}\right]}{\sin \left[\theta_{3}-\theta_{4}\right]} \\
-\frac{\sin \left[\theta_{2}\right]}{\sin \left[\theta_{1}-\theta_{2}\right]} & -\frac{\sin \left[\theta_{1}\right]}{\sin \left[\theta_{1}-\theta_{2}\right]} & -\frac{\cos \left[\theta_{4}\right]}{\sin \left[\theta_{3}-\theta_{4}\right]} & -\frac{\cos \left[\theta_{3}\right]}{\sin \left[\theta_{3}-\theta_{4}\right]} \\
\frac{\cos \left[\theta_{2}\right]}{\sin \left[\theta_{1}-\theta_{2}\right]} & \frac{\cos \left[\theta_{1}\right]}{\sin \left[\theta_{1}-\theta_{2}\right]} & -\frac{\sin \left[\theta_{4}\right]}{\sin \left[\theta_{3}-\theta_{4}\right]} & -\frac{\sin \left[\theta_{3}\right]}{\sin \left[\theta_{3}-\theta_{4}\right]}
\end{array}\right), \\
& N=\left(\begin{array}{cccccccc}
0 & 1 & 0 & 0 & -1 & 0 & 0 & 0 \\
0 & 0 & 1 & 0 & 0 & 0 & 0 & -1 \\
-1 & 0 & 0 & 0 & 0 & 1 & 0 & -1 \\
0 & 0 & 0 & -1 & -1 & 0 & 1 & 0
\end{array}\right)
\end{aligned}
$$

\section{Список литературы}

[1] Raussendorf R., Briegel H.J. // Phys. Rev. Lett. 2001. V. 86. P. $5188-5191$.

[2] Нильсен М., Чанг И. Квантовые вычисления и квантовая информация. М.: Мир. 2006. 824 с.; Nielsen M., Chuang I.; Quantum Computation and Quantum Information. Cambridge: Cambridge University Press. 2000.

[3] Raussendorf R., Wei T.-C. // Annual Review of Condensed Matter Physics 2012. V. 3. P. 239-261.

[4] Lloyd S., Braunstein S.L. // Phys. Rev. Lett. 1999. V. 82. P. 1784.

[5] Menicucci N.C., van Loock P., Weedbrook C., Gu M., Ralph T.C., Nielsen M.A. // Phys. Rev. Lett. 2006. V. 97. P. 110501.

[6] Yokoyama S. et al. // Nat. Photon. 2013. V. 7. P. 982-986.

[7] Sun L.-h., Chen Y.-q., Li G.-z. // Optics Express. 2012. V. 20. N 3. P. $3176-3191$.

[8] Milne D.F., Korolkova N.V. // Phys. Rev. A. 2012. V. 85. P. 032310.

[9] Houhou O., Aissaoui H., Ferraro A. // Phys. Rev. A. 2015. V. 92. P. 063843.
[10] Yukawa M., Ukai R., van Loock P., Furusawa A. // Phys. Rev. A. 2008. V. 78. P. 012301.

[11] Su X. et al. // Nat. Commun. 2013. V. 4. P. 2828.

[12] Menicucci N.C. // Phys. Rev. A. 2011. V. 83. P. 062314.

[13] Королев С.Б., Тихонов К.С., Голубева Т.Ю., Голубев Ю.М. // Опт. и спектр. 2017. Т. 123. № 3. С. 384-392; Korolev S.B., Tikhonov K.S., Golubeva T.Yu., Golubev Yu.M. // Opt. Spectrosc. 2017. V. 123. P. 411.

[14] Gu M., Weedbrook C., Menicucci N.C., Ralph T.C., van Loock P. // Phys. Rev. A. 2009. V. 79. P. 062318.

[15] van Loock P., Weedbrook C., Gu M. // Phys. Rev. A. 2007. V. 76. P. 032321.

[16] Gorshkov A.V., André A., Lukin M.D., Sorensen A.S. // Phys. Rev. A. 2007. V. 76. P. 033805.

[17] Nunn J. et al. // Phys. Rev. Lett. 2008. V. 101. P. 260502.

[18] Golubeva T., Golubev Yu., Mishina O., Bramati A., Laurat J., Giacobino E. // Phys. Rev. A. 2011. V. 83(5). P. 053810.

[19] Fleischhauer M., Imamoglu A., Marangos J.P. // Rev. Mod. Phys. 2005. V. 77. P. 633.

[20] Liu C., Dutton Z., Behroozi C.H., Hau L.V. // Nature. 2001. V. 409. P. 490.

[21] Phillips D.F., Fleischhauer A., Mair A., et al. // Phys. Rev. Lett. 2001. V. 86. P. 783.

[22] Novikova I., Walsworth R.L., Xiao Y. // Laser Photon. Rev. 2012. V. 6. P. 333.

[23] Choi K.S., Deng H., Laurat J., Kimble H.J. // Nature. 2008. V. 452. P. 67-71.

[24] Novikova I., Phillips N.B., Gorshkov A.V. // Phys. Rev. A. 2008. V. 78. P. 021802(R).

[25] Nunn J., et al. // Phys. Rev. A. 2007. V. 75. P. 011401(R).

[26] Самбурская К., Голубева Т., Голубев Ю., Giacobino E. // Опт. и спектр. 2011. Т. 110. № 5. C. 827; Samburskaya K., Golubeva T., Golubev Yu., Giacobino E. // Opt. Spectrosc. 2011. V. 110(5). P. 775.

[27] Gorshkov A.V., André A., Lukin M.D., Sorensen A.S. // Phys. Rev. A. 2007. V. 76. P. 033806.

[28] Reim K.F., Nunn J., Lorenz V.O., et al. // Nat. Photon. 2010. V. 4. P. 218.

[29] Golubeva T., Golubev Yu., Mishina O., Bramati A., Laurat J., Giacobino E. // Eur. Phys. J. D. 2012. V. 66. P. 275.

[30] Afzelius M., Simon C., de Riedmatten H., Gisin N. // Phys. Rev. A. 2009. V. 79. P. 052329.

[31] Jobez P., Usmani I., Timoney N., et al. // New J. Phys. 2014. V. 16. P. 083005.

[32] Saglamyurek E., Sinclair N., Slater J.A., et al. // New J. Phys. 2014. V. 16. P. 065019.

[33] Bonarota M., Le Gouët J.-L., Chaneliére T. // New J. Phys. 2011. V. 13. P. 013013.

[34] Gerasimov K.I., Moiseev S.A., Morozov V.I., Zaripov R.B. // SPIE Optical Technologies for Telecommunications 2015. V. 9533. P. 953310.

[35] Muschik C.A., Hammerer K., Polzik E.S., Cirac J.I. // Phys. Rev. A. 2006. V. 73. P. 062329.

[36] Julsgaard B., et al. // Nature. 2004. V. 432. P. 482.

[37] Zhang Y.-R., Yue J.-D., Fan H. // Phys. Rev. A. 2015. V. 91. P. 022104.

[38] Liang L., Lin G.W., Hao Y.M., Niu Y.P., Gong S.Q. // Phys. Rev. A. 2014. V. 90. P. 055801.

[39] Gorshkov A.V., Calarco T., Lukin M.D., Sorensen A.S. // Phys. Rev. A. 2008. V. 77. P. 043806.

[40] Manukhova A.D., Tikhonov K.S., Golubeva T.Yu., Golubev Yu.M. // Phys. Rev. A. 2017. V. 96. P. 023851. 
[41] Тихонов К.С., Голубева Т.Ю., Голубев Ю.М. // Опт. и спектр. 2015. Т. 118. № 5. С. 807-814; Tikhonov K.S., Golubeva T.Yu., Golubev Yu.M. // Opt. Spectrosc. 2015. V. 118. P. 773.

[42] Yadsan-Appleby H., Serafini A. // Phys. Lett. A. 2011. V. 375. P. 1864.

[43] Ukai R., Multi-Step Multi-Input One-Way Quantum Information Processing with Spatial and Temporal Modes of Light: Springer, Japan. 2015.

[44] Королев С.Б., Вашукевич Е.А., Голубева Т.Ю., Голубев Ю.М. // Квант. электрон. 2018. Т. 48. № 10. C. 906-911; Korolev S.B., Vashukevich E.A., Golubeva T.Yu., Golubev Yu.M. // Quant. Electron. 2018. V. 48(10). P. 906-911. 